\title{
ARANHAS DE SOLO (ARACHNIDA: ARANEAE) EM DIFERENTES FRAGMENTOS FLORESTAIS NO SUL DA BAHIA, BRASIL
}

\author{
Biota Neotropica v5 (n1)a-http://www.biotaneotropica.org.br/v5n1a/pt/abstract?inventory+BN010051a2005
}

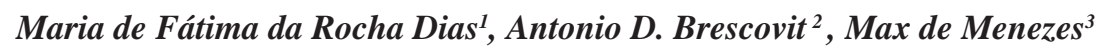

Recebido em 07/12/2003

Publicado em 01/02/2005

\begin{abstract}
1. Universidade do Estado da Bahia, Campus VII, Departamento de Educação, Laboratório de Ecologia de Artrópodes Terrestres. Rodovia Lomanto Júnior BR 407 Km 127, Senhor do Bonfim, Bahia, Brasil. 48970-000.

E-mail: faldias@yahoo.com.br

${ }^{2}$ Laboratório de Artrópodes. Instituto Butantan. Av. Vital Brazil, 1500. São Paulo.S.P. Brasil. 05503-900.

E-mail: anyphaenidae@butantan.gov.br

${ }^{3}$ Universidade Estadual de Santa Cruz. Rodovia Ilhéus/Itabuna, km16, NUPPE. Ilhéus.Bahia.Brasil. 45650-000.

E-mail: maxmz@uesc.br
\end{abstract}

\begin{abstract}
The southern area of the Atlantic Rainforest in the state of Bahia is suffering an accelerated fragmentation process by antropic action with the consequent alteration in the faunistic composition. The spiders are one of the dominant faunistic groups, and its importance for the stability of the ecosystems is unquestionable. The present research aim the investigating and comparing relative abundance and composition in species of soil spiders in the six environments forests: cocoa plantation in cabruca, capoeira, edge and interior of forest fragment, and edge and interior of continuous forest, located near the cities of Ilhéus and Una, state of Bahia. Six replicates were carried out in each environments, totalizing 36 sampling transects. In each transect 10 pitfall traps, with 6,0 cm opening diameter, were placed in straight lines, $15 \mathrm{~m}$ from each other. The preserving liquid used was formol $4 \%$ and the pitfall traps were left in the field for seven days. A total of four carried out between October/1999 and September/2000. The occurrence of 98 morphospecies, belonging to 38 genera and 30 families was verified. Of a total of the 2090 collected spiders only the 1450 adult specimens were used for the statistical analysis. The most abundant families were Salticidae (26,55\%), Oonopidae (20,07\%) and Pholcidae (7,03\%). In relation to species richness, the families that stood out were Salticidae (34,69\%), Oonopidae (9,18\%), Corinnidae and Ctenidae (5,1\%). It was not possible to detect differences between relative abundance and species composition of the sampled environments. However, the maintenance of the considered environments is important in order to preserve the araneofauna in the southern area of Bahia, once they represent the local landscape.
\end{abstract}

Key words: Atlantic Forest, spiders, soil, Neotropical, pitfall traps..

\section{Resumo}

A faixa de Mata Atlântica do Sul da Bahia vem sofrendo acelerado processo de fragmentação por ação antrópica, com conseqüente alteração da sua composição faunística. As aranhas constituem um dos grupos predadores dominantes no solo de florestas e sua importância para a estabilidade dos ecossistemas é indiscutível. A pesquisa teve o objetivo de comparar abundância relativa e composição em espécies de aranhas de solo em 6 ambientes florestais: cacaual em cabruca, capoeira, borda e interior de fragmento de mata e borda e interior de mata contínua, localizados nos municípios de Ilhéus e Una. Para cada um dos ambientes analisados existiam um total de seis repetições, totalizando 36 transectos. As aranhas foram amostradas em transectos, em cada um, foram utilizadas 10 armadilhas de queda "pitfall” com diâmetro de abertura 6,0 cm, posicionadas em linha, a cada $15 \mathrm{~m}$. O conservante utilizado foi formol a 4\% e o tempo de permanência das armadilhas no campo foi de sete dias. No total, foram realizadas quatro coletas entre outubro de 1999 e setembro de 2000. Foram coletadas 2090 aranhas, das quais apenas 1450 espécimes adultos e estes foram os utilizados nas análises estatísticas. Entre os adultos foi constatada a ocorrência de 98 morfoespécies, pertencentes a 38 gêneros e 30 famílias. As famílias com maior abundância de espécimens foram Salticidae (26,55\%), Oonopidae (20,07\%) e Pholcidae (7,03\%). Em relação à riqueza de morfoespécies, destacaram-se as famílias Salticidae (34,69\%), Oonopidae (9,18\%), Ctenidae e Corinnidae com (5,1\%). Da forma como a pesquisa foi conduzida não foi possível detectar diferenças na composição e abundâncias de aranhas de solo. No entanto, a manutenção dos ambientes considerados é importante para a preservação da araneofauna da região Sul da Bahia, uma vez que eles representam a paisagem local.

Palavras-chave: floresta atlântica, aranhas, solo, Neotropical. 


\section{Introdução}

A Mata Atlântica é hoje representada por remanescentes florestais com diferentes níveis de perturbação antrópica, geralmente isolados entre si. Esta redução de sua área original, conhecida como fragmentação de hábitat, torna a dinâmica das comunidades biológicas diferentes daquela prevista para sistemas naturais contínuos (Myers 1980, Noss \& Csuti 1994, Dean 1996, Laurance \& Bierregaard 1997).

A região Sul da Bahia é composta de florestas úmidas caracterizadas por árvores altas, com folhas sempre verdes e abundância de epífitas, é considerada um sistema florestal com elevada riqueza de espécies e alto grau de endemismo. No entanto, a existência dessa expressiva riqueza não tem sido suficiente para evitar a destruição insensata que tem afetado gravemente esses ambientes (Araujo et al. 1998)

Os artrópodes proporcionam numerosas oportunidades para investigações sobre comunidades ecológicas devido ao seu pequeno tamanho, abundância, importância no ciclo de nutrientes e fluxo de energia nos ecossistemas (Uetz 1976). Dentre a enorme diversidade de artrópodes, as aranhas são um dos grupos mais abundantes e, a exemplo de artrópodes de solo de florestas, estão envolvidas em processos essenciais no ecossistema, tais como as transferências de energia nas cadeias alimentares (Roth 1993).

Segundo Turnbull (1973), o estudo da composição das comunidades de aranhas tem objetivo principal de reunir dados taxonômicos e estatísticos que vão caracterizar essas comunidades em determinado ambiente. As informações contidas nesses estudos constituem, de maneira geral, a base para a realização de estudos mais complexos em ecologia.

Até o presente, nenhum trabalho específico a respeito da fauna araneológica de solo foi realizado nessa área. Torna-se necessário iniciar estudos ecológicos que permitam uma real caracterização desse ecossistema, bem como registrar a memória dos seus recursos naturais antes que se percam nesse rápido processo de desmatamento que vem ocorrendo nesta região. Desta forma esta pesquisa teve o objetivo de comparar abundância relativa e composição em espécies de aranhas de solo em ambientes florestais no Sul da Bahia com diferentes graus de perturbação antrópica.

\section{Material e Métodos}

A área de estudo esta compreendida entre as coordenadas $14^{\circ} 48^{\prime} 00^{\prime \prime} \mathrm{S}$; $39^{\circ} 02^{\prime} 00^{\prime \prime} \mathrm{W}$ e $15^{\circ} 17^{\prime} 48^{\prime \prime} \mathrm{S}$; 3904'28’'W, nos municípios de Ilhéus e Una no Sul da Bahia. A pesquisa foi realizada de forma a permitir comparação entre seis ambientes florestais com diferentes graus de perturbação antrópica abaixo caracterizados. Para cada um dos ambientes analisados existiam um total de seis réplicas, totalizando 36 transectos.
- interior de mata contínua (ICO), constituído por áreas de floresta madura, com mais de 1000 ha a mais de 100 $m$ de qualquer borda.

- borda de mata contínua (BCO), áreas com mais de 1000 ha, a menos de $20 \mathrm{~m}$ da área de contato com pastagem;

- interior de fragmento de mata (IFR), formado por áreas de mata madura com menos de 100 ha, a mais de 100 m de qualquer borda;

- borda de fragmento de mata, áreas com menos de 100 ha, a menos de $20 \mathrm{~m}$ da área de contato com pastagem;

- cabruca (CB), plantações de cacau (Theobroma cacao) implantadas pela substituição do sub-bosque por cacaueiros, mantendo o sombreamento com espécies arbóreas da floresta original previamente selecionadas, com 30 ha ou mais;

- capoeira (CP), formada por vegetação florestal secundária em estágio sucessional inicial, com 30 ha ou mais.

\section{Coletas}

As coletas foram feitas em quatro períodos entre outubro de 1999 e setembro de 2000. Para cada ambiente foram utilizados seis transectos de 150 metros. Utilizaramse 10 armadilhas de solo do tipo "pitfall" com capacidade de 500 ml e diâmetro de abertura $6,0 \mathrm{~cm}$, colocadas paralelamente e a aproximadamente $5 \mathrm{~m}$ dos transectos, distando uma da outra 15 metros. Cada armadilha possuía uma cobertura, feita de prato plástico com $15 \mathrm{~cm}$ de diâmetro, elevada a cerca de $5 \mathrm{~cm}$ do chão, suportado por hastes de madeira. $\mathrm{O}$ líquido conservante utilizado foi formol a $4 \%$. As armadilhas foram deixadas no campo e retiradas sete dias após sua colocação e levadas ao laboratório da Universidade Estadual de Santa Cruz para a triagem e identificação do material.

\section{Identificação do material}

A identificação das famílias foi feita através da chave dicotômica de Pikelin \& Schiapelli (1963). A nomenclatura obedeceu a do catálogo de Platnick (2005). O nível taxonômico foi específico ou, na impossibilidade de atingilo, os exemplares ficaram registrados como morfoespécies. A confirmação da identificação foi feita pelos pesquisadores Dr. Antonio D. Brescovit (Instituto Butantan) para Araneomorphae e Dr. Rogério Bertani (Instituto Butantan) para Mygalomorphae. Para fins qualitativos os espécimes jovens foram identificados em família, não sendo incluídos nas análises estatísticas. O material foi depositado na Coleção de Aracnídeos do Laboratório de Artrópodes do Instituto Butantan (São Paulo; A. D. Brescovit, curador). 


\section{Análise dos dados}

Espécies raras: foram selecionadas as morfoespécies cuja abundância relativa foi maior que $100 / \mathrm{S}$, onde S se refere ao número total de morfoespécies encontradas nos ambientes amostrados. Desta forma, foram excluídas as morfoespécies raras (Richardson et al. 1980). Além disto, análises ecológicas baseadas em matrizes muito grandes, devido à inclusão de taxa que ocorrem com freqüência esporádica, fornecem resultados duvidosos, pois se atribui alta correlação à taxa que possuem grande número de ausência simultânea.

Análise de componentes principais (PCA): Análise indireta no qual as amostras e/ou espécies são plotadas ao longo de eixos de variação da composição da comunidade ou alteração do espaço/tempo, os quais podem, subseqüentemente, ser interpretadas em termos de gradiente ambiental. Foi utilizado o programa CANOCO 4.0 (Terbraak et al. 1998)

Para comparação da composição de espécies entre os ambientes foi utilizado o teste de Anova no Programa Biostat 2.0 (Ayres et al. 2000). Para análise de agrupamento-Técnica hierárquica baseada no grau de similaridade entre as amostras. Foi utilizado o programa MVSP 3.0, onde a forma de agrupar foi UPGMA com percentual de similaridade de Jaccard.

\section{Resultados}

Foram coletadas 2090 aranhas, sendo 1450 adultas, distribuídas em 30 famílias e 98 morfoespécies (Tabela 1). Do total de morfoespécies coletadas, 3 foram identificadas até espécie, 38 até gênero. As morfoespécies estão distribuídas em 6 famílias de Mygalomorphae e 24 de Araneomorphae. Considerando-se o número total de indivíduos coletados por família, constata-se que Salticidae (544indivíduos) foi a mais abundante na Região de Una, seguida de Oonopidae (321), Pholcidae (158), Ctenidae (156), Ochyroceratidae (136) e Zodariidae (123), e as menos abundantes foram Theridiosomatidae, Pisauridae, Sparassidae, Anapidae, Barychelidae, Caponiidae, Gnaphosidae e Symphytognathidae, todas com no máximo três indivíduos (Figura 1). Em relação ao número de morfoespécies, a família mais rica foi Salticidae (34 morfoespécies), seguida de Oonopidae (9), Ctenidae, Coriniidae e Theridiidae com (5), Linyphiidae (4), as restantes representadas por 3 morfoespécies ou menos (Figura 2).

Dentre as 98 morfoespécies encontradas apenas 27 apresentaram abundâncias maiores que 1,02 \%, as mais abundantes foram Neoxyphinus sp. 1 (8,35\%), Salticidae sp. 1 (6,62\%), Tenedos sp. 1 (6,07\%), Gamasomorphinae sp. $1(6,07 \%)$ e Pholcidae sp. 1 (5,65\%) que ocorrem em todos os ambientes, e Ochyrocera sp. 1 (6,07\%), abundante em todos os ambientes a exceção de capoeira. As demais apresentaram abundâncias menores que $4 \%$.

\section{Análise de Componentes Principais (PCA)}

Através da Análise de Componentes Principais, obteve-se 58,6\% de explicação da variação encontrada, através das interpretações dos eixos I e II, sendo que o primeiro eixo com $37,2 \%$ esta relacionado com a perturbação dos ambientes estudados e dão destaque positivo aos ambientes menos alterados neste estudo, como: interior de mata contínua, borda de mata contínua, interior de fragmento de mata e borda de fragmento de mata, e, negativamente para cabruca e capoeira dois ambientes alterados antrópicamente. O segundo eixo com 21,4\% plota as espécies a conduções de simplificação da mata, ou seja, os ambientes que tiveram a vegetação original removida. A analisando a figura 3, constata-se que:

- as morfoespécies aff. Bolostromus sp., Linyphiidae sp. 1, Hahniidae sp. 1, Salticidae sp. 1, Linothele sp., Celaetychaeus sp., Salticidae sp. 12, Salticidae sp. 23 e Orchestina sp. 1, apresentam maiores abundâncias nos ambientes de mata continua e queda de abundância nos ambientes de cabruca e capoeira;

- morfoespécies como Ochyrocera sp. 1, Salticidae sp. 10 e Ianduba sp., também apresentam maiores abundâncias nos ambientes de mata continua, mas já crescem em abundâncias nos fragmentos;

- as morfoespécies Blechroscelis sp., Otiothops atlanticus , Mysmenidae sp. 1, Salticidae sp. 9, Pholcidae sp. 1 e Lygromma sp. 2 apresentam maiores abundâncias nos ambientes de fragmentos de mata;

- As morfoespécies Tenedos sp. 1 e Oligoxystre sp. parecem não sofrer efeitos da perturbação (retas muito pequenas e no centro do diagrama);

- As morfoespécies Lygromma sp. 1, Gamasomorphinae sp. 2, Alpaida sp., Neoxyphinus sp. 1, aff. Neonella sp. e Gamasomorphinae sp. 1 apresentam maiores abundâncias nos ambientes de cabruca e capoeira.

\section{Análise de agrupamento}

A análise do dendrograma de similaridade de espécies (Figura 4) mostrou a formação de quatro grupos ao nível de corte a 95,2\%, onde capoeira, cabruca e interior de mata contínua ficam isolados, o interior de fragmento de mata liga-se ao grupo das bordas de mata contínua e dos fragmentos de mata, estes últimos apresentado maior similaridade entre si.

\section{Discussão}

Devido ao pouco conhecimento da fauna de aranhas de solo, tivemos grande dificuldade na identificação das espécies. Dentre as morfoespécies não identificadas, as morfoespécies de Hahniidae, Oonopidae, Nothrocteneus, Pseudanapis, Masteria e Ochyrocera, certamente são espécies novas, mas este número pode ser muito maior. Para 


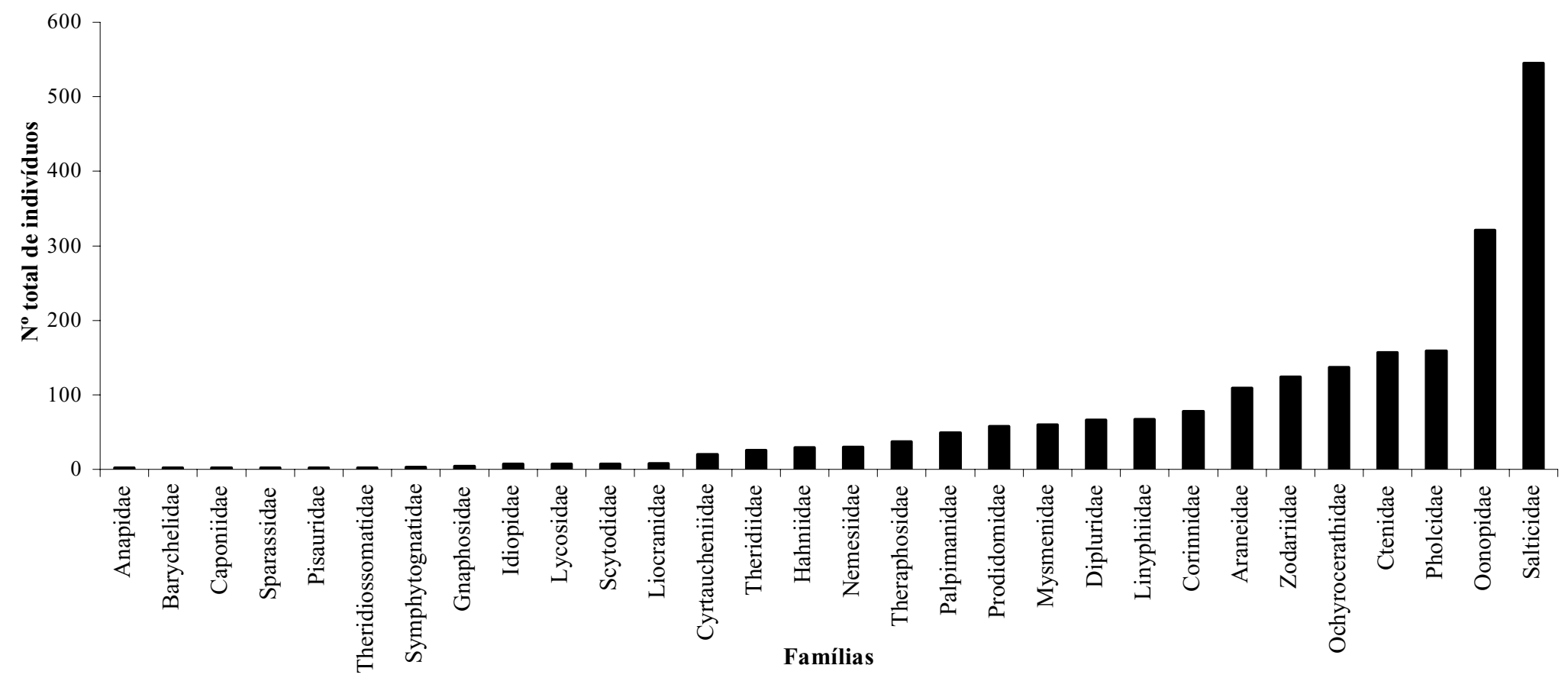

Figura 1. Distribuição do número total de indivíduos coletados por família nos seis ambientes amostrados da Região Sul da Bahia

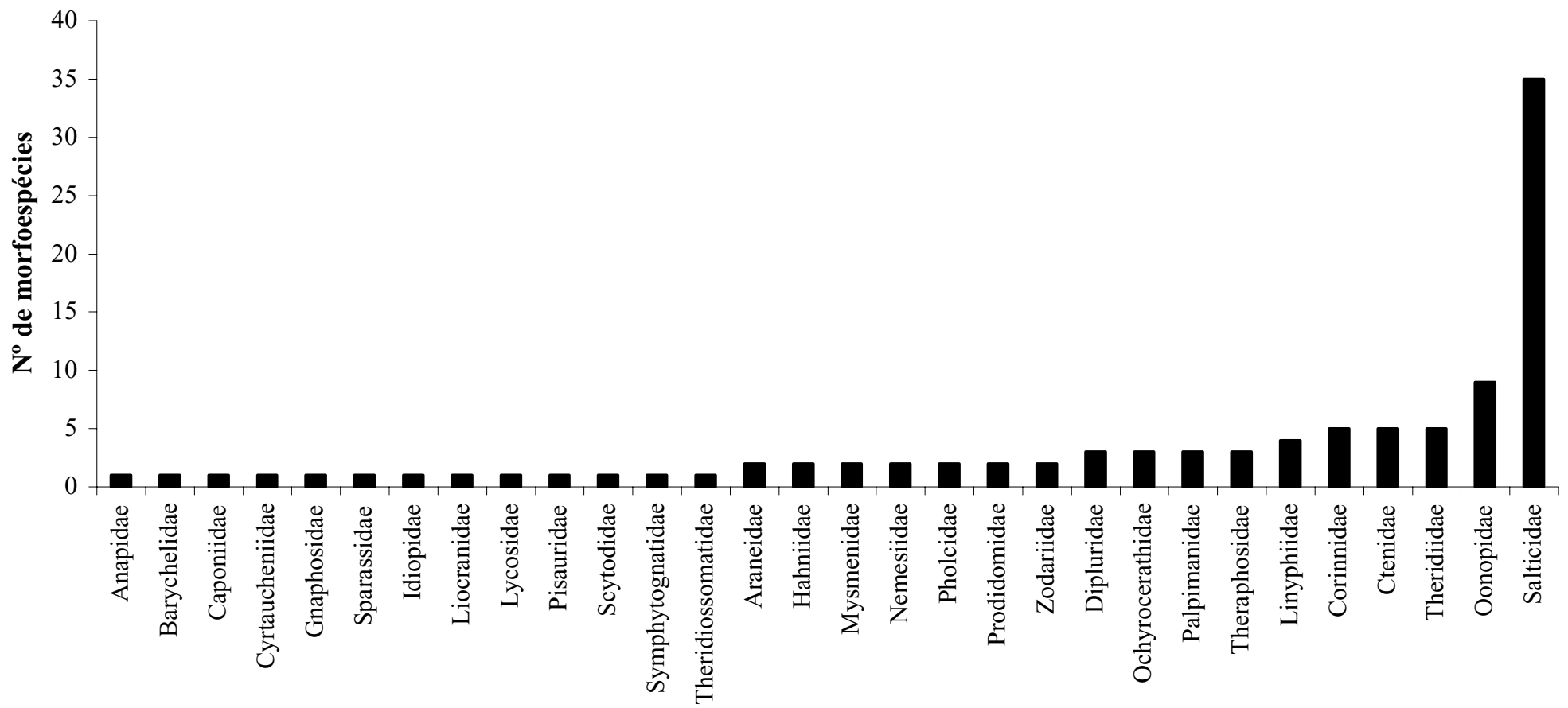

Famílias

Figura 2. Distribuição do número total de morfoespécies por família nos seis ambientes amostrados da Região Sul da Bahia. 


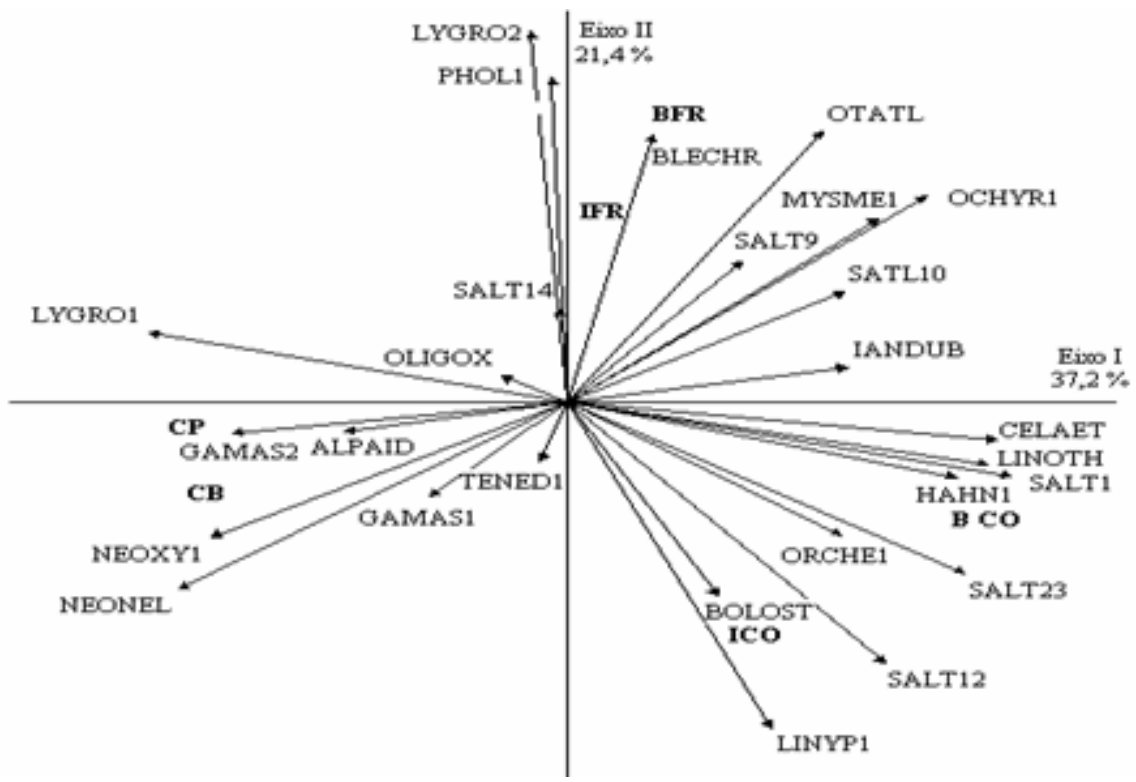

Figura 3. Análise de componentes principais de aranhas na Região Sul da Bahia.

UPGMA
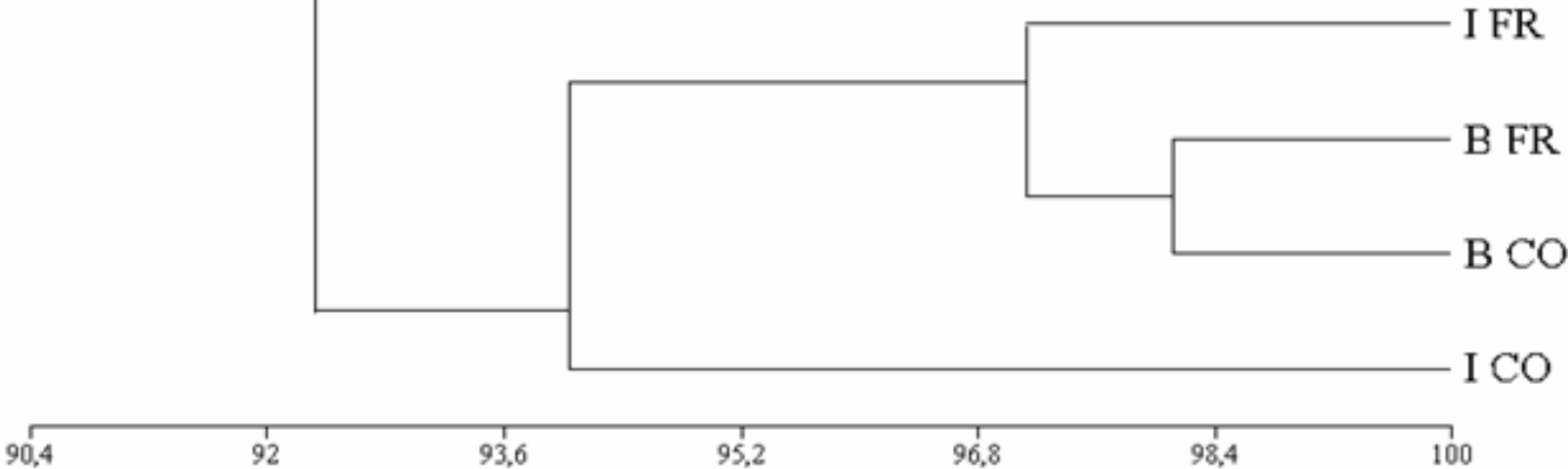

percentual de sireilaridsde

Figura 4. Análise de agrupamento para os ambientes amostrados na Região Sul da Bahia, calculada a partir da similaridade de Jaccard e agrupado pelo método UPGMA.

ICO - Interior de mata contínua;

BCO - Borda de mata contínua;

IFR - Interior de fragmento de mata;

BFR - Borda de fragmento de mata;

$C B$ - Cabruca;

$C P$ - Capoeira

http://www.biotaneotropica.org.br 
Tabela I - Ocorrência e abundância relativa das morfoespécies nos seis ambientes amostrados na Região Sul da Bahia.

\begin{tabular}{|c|c|c|c|c|c|c|c|c|c|}
\hline \multirow[b]{2}{*}{ Família } & \multirow[b]{2}{*}{ Morfoespécies } & \multicolumn{6}{|c|}{ AMBIENTES } & \multirow[b]{2}{*}{$\begin{array}{l}\mathbf{N}^{\mathbf{o}} \\
\text { Ind. }\end{array}$} & \multirow[b]{2}{*}{$\begin{array}{l}\text { Ab. Rel. } \\
(\%)\end{array}$} \\
\hline & & ICO & BCO & IFR & BFR & $\mathbf{C B}$ & $\mathbf{C P}$ & & \\
\hline Anapidae & Pseudanapis sp. & 0 & 0 & 0 & 1 & 0 & 0 & 1 & 0,07 \\
\hline Araneidae & Araneidae sp. & 4 & 2 & 0 & 1 & 1 & 1 & 9 & 0,69 \\
\hline Araneidae & Alpaida sp. & 2 & 3 & 3 & 2 & 2 & 7 & 19 & 1,31 \\
\hline Barychelidae & Sasoninae sp. & 0 & 0 & 1 & 0 & 0 & 0 & 1 & 0,07 \\
\hline Caponiidae & Nops sp. & 0 & 0 & 1 & 0 & 0 & 0 & 1 & 0,07 \\
\hline \multirow[t]{5}{*}{ Corinnidae } & Falconina sp. & 0 & 0 & 1 & 0 & 1 & 0 & 2 & 0,14 \\
\hline & Corinnidae sp. & 1 & 1 & 2 & 0 & 0 & 0 & 4 & 0,28 \\
\hline & Castianeira sp. & 4 & 3 & 1 & 0 & 1 & 3 & 12 & 0,83 \\
\hline & Ianduba sp. & 5 & 9 & 2 & 9 & 4 & 4 & 33 & 2,28 \\
\hline & Mymercium sp. & 1 & 0 & 0 & 1 & 0 & 0 & 2 & 0,14 \\
\hline \multirow[t]{5}{*}{ Ctenidae } & Ctenus sp. & 1 & 0 & 0 & 0 & 0 & 0 & 1 & 0,07 \\
\hline & Isoctenus sp. & 1 & 0 & 1 & 0 & 0 & 0 & 2 & 0,14 \\
\hline & Nothroctenus sp. & 0 & 1 & 1 & 3 & 0 & 0 & 6 & 0,41 \\
\hline & Ctenidae sp. & 1 & 0 & 3 & 1 & 2 & 0 & 7 & 0,48 \\
\hline & Celaetychaeus sp. & 9 & 8 & 6 & 7 & 4 & 2 & 36 & 2,48 \\
\hline Cyrtauchenidae & aff. Bolostromus sp. & 3 & 6 & 3 & 1 & 5 & 1 & 19 & 1,31 \\
\hline \multirow{3}{*}{ Dipluridae } & Dipluridae sp. & 1 & 3 & 1 & 2 & 0 & 1 & 8 & 0,55 \\
\hline & Masteria sp. & 3 & 4 & 1 & 1 & 1 & 1 & 11 & 0,76 \\
\hline & Linothele sp. & 7 & 5 & 3 & 4 & 0 & 0 & 19 & 1,31 \\
\hline Gnaphosidae & Gnaphosidae sp. & 0 & 0 & 0 & 0 & 2 & 0 & 2 & 0,14 \\
\hline \multirow[t]{2}{*}{ Hahniidae } & Hahniidae sp. 1 & 3 & 11 & 2 & 3 & 0 & 1 & 20 & 1,38 \\
\hline & Hahniidae sp. 2 & 0 & 1 & 0 & 0 & 0 & 3 & 4 & 0,28 \\
\hline \multirow[t]{4}{*}{ Linyphiidae } & Brattia sp. & 0 & 0 & 0 & 0 & 0 & 1 & 1 & 0,07 \\
\hline & Linyphiidae sp. 1 & 7 & 5 & 10 & 10 & 2 & 3 & 37 & 2,55 \\
\hline & Linyphiidae sp. 2 & 0 & 0 & 0 & 0 & 9 & 1 & 10 & 0,69 \\
\hline & Linyphiidae sp. 3 & 0 & 0 & 0 & 0 & 3 & 0 & 3 & 0,21 \\
\hline Liocranidae & Orthobula sp. & 0 & 1 & 1 & 0 & 1 & 1 & 4 & 0,28 \\
\hline Lycosidae & Lycosidae sp. & 0 & 0 & 0 & 0 & 2 & 1 & 3 & 0,21 \\
\hline \multirow[t]{2}{*}{ Mysmenidae } & Mysmenidae sp. 1 & 0 & 9 & 6 & 6 & 0 & 20 & 2 & 37 \\
\hline & Mysmenidae sp. 2 & 0 & 0 & 2 & 0 & 4 & 2 & 8 & 0,55 \\
\hline Nemesiidae & Rachias sp. & 0 & 3 & 1 & 1 & 4 & 3 & 12 & 0,83 \\
\hline \multirow[t]{3}{*}{ Ochyrocerathidae } & Ochyrocera sp. 2 & 0 & 0 & 4 & 1 & 0 & 0 & 5 & 0,34 \\
\hline & Theotima sp. & 0 & 2 & 0 & 1 & 4 & 0 & 7 & 0,48 \\
\hline & Ochyrocera sp. 1 & 15 & 13 & 17 & 22 & 20 & 1 & 88 & 6,07 \\
\hline \multirow[t]{9}{*}{ Oonopidae } & Gamasomorphinae sp. 1 & 20 & 18 & 6 & 6 & 33 & 5 & 88 & 6,07 \\
\hline & Gamasomorphinae sp. 2 & 6 & 0 & 2 & 6 & 8 & 6 & 28 & 1,93 \\
\hline & Gamasomorphinae sp. 3 & 1 & 2 & 1 & 0 & 0 & 0 & 4 & 0,28 \\
\hline & Orchestina sp. 1 & 6 & 2 & 3 & 3 & 8 & 7 & 29 & 2,00 \\
\hline & Orchestina sp. 2 & 2 & 0 & 0 & 3 & 1 & 2 & 8 & 0,55 \\
\hline & Orchestina sp. 3 & 1 & 0 & 0 & 0 & 1 & 0 & 2 & 0,14 \\
\hline & Neoxyphinus sp. 1 & 18 & 18 & 21 & 13 & 23 & 28 & 121 & 8,34 \\
\hline & Neoxyphinus sp. 2 & 0 & 2 & 2 & 2 & 2 & 1 & 9 & 0,69 \\
\hline & Neoxyphinus sp. 3 & 0 & 0 & 0 & 0 & 0 & 1 & 1 & 0,07 \\
\hline
\end{tabular}


Tabela I - Ocorrência e abundância relativa das morfoespécies nos seis ambientes amostrados na Região Sul da Bahia.

\begin{tabular}{|c|c|c|c|c|c|c|c|c|c|}
\hline \multirow[b]{2}{*}{ Família } & \multirow[b]{2}{*}{ Morfoespécies } & \multicolumn{6}{|c|}{ AMBIENTES } & \multirow[b]{2}{*}{$\begin{array}{l}\mathbf{N}^{\circ} \\
\text { Ind. }\end{array}$} & \multirow[b]{2}{*}{$\begin{array}{c}\text { Ab. Rel. } \\
\text { (\%) }\end{array}$} \\
\hline & & $\begin{array}{r}\text { IC } \\
\mathrm{C}\end{array}$ & BCO & IFR & BFR & CB & $\mathbf{C P}$ & & \\
\hline \multirow[t]{3}{*}{ Palpimanidae } & Otiothops atlanticus & 4 & 6 & 7 & 8 & 1 & 5 & 31 & 2,14 \\
\hline & Otiothops aff. gounellei & 2 & 0 & 0 & 0 & 1 & 0 & 3 & 0,21 \\
\hline & Fernandezina sp. & 2 & 2 & 1 & 1 & 0 & 1 & 8 & 0.48 \\
\hline \multirow[t]{2}{*}{ Pholcidae } & Blechroscelis sp. & 0 & 10 & 0 & 9 & 0 & 1 & 20 & 1,38 \\
\hline & Pholcidae sp. 1 & 10 & 20 & 19 & 14 & 9 & 10 & 82 & 5,65 \\
\hline \multirow[t]{2}{*}{ Prodidomidae } & Lygromma sp. 1 & 3 & 1 & 4 & 3 & 4 & 5 & 20 & 1,38 \\
\hline & Lygromma sp. 2 & 11 & 5 & 6 & 4 & 2 & 3 & 31 & 2,14 \\
\hline \multirow[t]{34}{*}{ Salticidae } & aff. Neonella sp. & 0 & 10 & 5 & 8 & 3 & 2 & 28 & 1,93 \\
\hline & Scopocera sp. & 0 & 1 & 0 & 1 & 0 & 0 & 2 & 0,14 \\
\hline & Formiciformes sp. & 0 & 1 & 0 & 1 & 1 & 2 & 5 & 0,34 \\
\hline & Salticidae sp. 1 & 19 & 19 & 15 & 17 & 13 & 13 & 96 & 6,62 \\
\hline & Salticidae sp. 2 & 1 & 3 & 3 & 1 & 0 & 2 & 10 & 0,69 \\
\hline & Salticidae sp. 3 & 0 & 1 & 0 & 2 & 0 & 0 & 3 & 0,21 \\
\hline & Salticidae sp. 4 & 2 & 0 & 0 & 0 & 2 & 1 & 5 & 0,34 \\
\hline & Salticidae sp. 5 & 1 & 0 & 1 & 0 & 0 & 0 & 2 & 0,14 \\
\hline & Salticidae sp. 7 & 1 & 0 & 0 & 1 & 5 & 5 & 12 & 0,83 \\
\hline & Salticidae sp. 8 & 0 & 3 & 2 & 1 & 0 & 3 & 9 & 0,69 \\
\hline & Salticidae sp. 9 & 9 & 8 & 12 & 8 & 7 & 7 & 51 & 3,52 \\
\hline & Salticidae sp. 10 & 8 & 12 & 16 & 7 & 2 & 7 & 52 & 3,59 \\
\hline & Salticidae sp. 11 & 1 & 0 & 0 & 0 & 1 & 1 & 3 & 0,21 \\
\hline & Salticidae sp. 12 & 12 & 9 & 1 & 2 & 1 & 2 & 27 & 1,86 \\
\hline & Salticidae sp. 13 & 0 & 1 & 0 & 0 & 1 & 0 & 2 & 0,14 \\
\hline & Salticidae sp. 14 & 2 & 2 & 1 & 5 & 5 & 0 & 15 & 1,03 \\
\hline & Salticidae sp. 15 & 0 & 4 & 0 & 1 & 6 & 0 & 11 & 0,76 \\
\hline & Salticidae sp. 16 & 2 & 0 & 0 & 0 & 0 & 0 & 2 & 0,14 \\
\hline & Salticidae sp. 17 & 0 & 0 & 0 & 0 & 1 & 0 & 1 & 0,07 \\
\hline & Salticidae sp. 18 & 0 & 2 & 0 & 0 & 0 & 1 & 3 & 0,21 \\
\hline & Salticidae sp. 19 & 0 & 1 & 0 & 3 & 2 & 4 & 10 & 0,69 \\
\hline & Salticidae sp. 20 & 0 & 0 & 0 & 0 & 1 & 0 & 1 & 0,07 \\
\hline & Salticidae sp. 21 & 1 & 0 & 1 & 0 & 0 & 1 & 3 & 0,21 \\
\hline & Salticidae sp. 22 & 1 & 0 & 0 & 0 & 0 & 0 & 1 & 0,07 \\
\hline & Salticidae sp. 23 & 4 & 7 & 2 & 1 & 1 & 0 & 15 & 1,03 \\
\hline & Salticidae sp. 24 & 0 & 1 & 0 & 0 & 0 & 0 & 1 & 0,07 \\
\hline & Salticidae sp. 25 & 0 & 0 & 0 & 0 & 1 & 0 & 1 & 0,07 \\
\hline & Salticidae sp. 26 & 0 & 0 & 0 & 0 & 0 & 1 & 1 & 0,07 \\
\hline & Salticidae sp. 27 & 0 & 0 & 0 & 1 & 0 & 0 & 1 & 0,07 \\
\hline & Salticidae sp. 28 & 0 & 1 & 1 & 0 & 0 & 0 & 2 & 0,14 \\
\hline & Salticidae sp. 29 & 2 & 0 & 0 & 0 & 0 & 0 & 2 & 0,14 \\
\hline & Salticidae sp. 30 & 0 & 0 & 0 & 0 & 1 & 1 & 2 & 0,14 \\
\hline & Salticidae sp. 31 & 0 & 1 & 0 & 2 & 0 & 0 & 3 & 0,21 \\
\hline & Salticidae sp. 32 & 0 & 0 & 0 & 1 & 2 & 0 & 3 & 0,21 \\
\hline Scytodidae & Scytodes iabaday & 1 & 0 & 0 & 2 & 2 & 0 & 5 & 0,34 \\
\hline Sparassidae & Sparianthinae sp. & 0 & 0 & 0 & 0 & 0 & 1 & 1 & 0,07 \\
\hline Symphytognatl & Anapistula sp. & 0 & 1 & 1 & 0 & 0 & 0 & 2 & 0,14 \\
\hline
\end{tabular}


Tabela I - Ocorrência e abundância relativa das morfoespécies nos seis ambientes amostrados na Região Sul da Bahia.

\begin{tabular}{|c|c|c|c|c|c|c|c|c|c|}
\hline \multirow[b]{3}{*}{ Família } & \multirow[b]{3}{*}{ Morfoespécies } & \multicolumn{8}{|c|}{ conclusão } \\
\hline & & \multicolumn{6}{|c|}{ AMBIENTES } & \multirow[b]{2}{*}{$\begin{array}{l}\mathbf{N}^{0} \\
\text { Ind. }\end{array}$} & \multirow[b]{2}{*}{$\begin{array}{l}\text { Ab. Rel. } \\
(\%)\end{array}$} \\
\hline & & I CO & BCO & I FR & B FR & CB & $\mathbf{C P}$ & & \\
\hline \multirow[t]{3}{*}{ Theraphosidae } & Homeomma sp. & 1 & 0 & 1 & 0 & 0 & 0 & 2 & 0,14 \\
\hline & Theraphosidae sp. & 0 & 0 & 0 & 1 & 0 & 0 & 1 & 0,07 \\
\hline & Oligoxystre sp. & 6 & 1 & 3 & 5 & 2 & 5 & 22 & 1,52 \\
\hline \multirow[t]{5}{*}{ Theridiidae } & Styposis sp. & 0 & 2 & 1 & 0 & 8 & 0 & 11 & 0,76 \\
\hline & Achaearanea sp. & 0 & 0 & 0 & 0 & 0 & 1 & 1 & 0,07 \\
\hline & Dipoena sp. & 0 & 0 & 1 & 0 & 0 & 0 & 1 & 0,07 \\
\hline & Episinus sp. & 0 & 1 & 0 & 0 & 0 & 0 & 1 & 0,07 \\
\hline & Theridiidae sp. & 2 & 0 & 0 & 0 & 1 & 0 & 3 & 0,21 \\
\hline Theridiosomatidae & Theriosomatidae sp. & 0 & 0 & 0 & 0 & 1 & 0 & 1 & 0,07 \\
\hline \multirow[t]{2}{*}{ Zodariidae } & Tenedos sp. 1 & 28 & 10 & 7 & 19 & 18 & 6 & 88 & 6,07 \\
\hline & Tenedos sp. 2 & 1 & 1 & 0 & 1 & 0 & 0 & 3 & 0,21 \\
\hline TOTAL & & 259 & 279 & 219 & 240 & 253 & 200 & 1450 & 100 \\
\hline
\end{tabular}

a maioria dos grupos, a literatura disponível simplesmente não permite determinar se as aranhas coletadas correspondem a espécies que já foram ou não descritas, uma vez que estes não foram revisados, e as descrições e ilustrações publicadas não são adequadas para o reconhecimento das espécies.

A região Sul da Bahia é um bom exemplo de área ainda mal conhecida cientificamente em relação às aranhas. É considerada um sistema florestal com elevada riqueza de espécies e alto grau de endemismo para plantas (Mori et al. 1981, Prance 1982), insetos (Brown 1991), répteis (Jackson 1978), aves (Haffer 1974) e mamíferos (Rylands 1982). Pelo menos dois casos de endemismos são conhecidos para aranhas da família Ctenidae: Phoneutria bahiensis, descrita por Simo \& Brescovit (2001) restrita até o momento para a região de Ilhéus e Una e de aranhas do gênero Celaetychaeus, descrito por Simon (1897), para o Sul da Bahia, do qual pelo menos 7 espécies são conhecidas (A.D.Brescovit, observ. pess.).

A família Salticidae foi a mais abundante no solo dos ambientes amostrados, contrariando os resultados obtidos em outros locais. Para a América do Norte, foram registradas em florestas as famílias Lycosidae, Gnaphosidae, Thomisidae, Clubionidae, Hahniidae, Theridiidae, Agelenidae e Linyphiidae como sendo as dominantes do solo (Uetz 1976, 1979, Post III \& Riechert 1977, Abraham, 1983, Corey \& Taylor 1988). Para uma floresta da Finlândia a família Linyphiidae foi a mais abundante (Palmgren \& Biström 1979). Embora já tenha sido registrado por Gasnier et al. (1995), Green (1999) e Toti et al. (2000), a grande abundância da família Salticidae, porém nas partes aéreas de árvores. Segundo Jocqué (1984), a família Salticidae é mais abundante em solo de regiões tropicais, uma vez que as altas temperaturas tropicais favorecem as aranhas de grande mobilidade, esta pode ser uma explicação para a grande abundância desta família nas amostras, além ser uma das maiores famílias em número de espécies descritas.

Na maioria das comunidades, umas poucas espécies são abundantes e muitas são raras (Odum 1985, Ricklefs 1996). Em relação à abundância das morfoespécies, apenas 27 (27,5\%) atingiram valores maiores que 1,02\% (100/S), enquanto que 71 (72,5\%) apresentaram abundância menor. Esse fato se deve ao ajuste entre os organismos no seu ambiente, definido pelo grau de adaptação de cada espécie (Pianka 1982, Odum 1985, Ricklefs 1996). A adaptação ocorre em várias dimensões, onde os organismos devem se encaixar simultaneamente aos padrões de temperatura, umidade, competidores, predadores, respeitando os seus limites. Assim sendo, as 71 morfoespécies pouco freqüentes nas coletas podem se acomodar nos ambientes estáveis e sem mudanças bruscas. O fato de muitas espécies de aranhas serem raras tem implicações para conservação. Se estas espécies de fato preferem estes ambientes, manter um mosaico de tipos de vegetação pode ser muito importante para a manutenção da diversidade total de aranhas da região. A raridade destas espécies nas amostras apenas aumenta a necessidade de manutenção destes ambientes para sua conservação.

As modificações físicas do ambiente alterado são responsáveis por muitas das modificações observadas nas comunidades de animais e plantas, bem como na biologia de muitos desses organismos. Assim, a probabilidade de ocorrência e manutenção de diversas espécies pode ser afetada direta ou indiretamente pelas modificações microclimáticas. Essas modificações se por um lado podem ser desfavoráveis a espécies adaptadas ao interior da mata, podem beneficiar espécies adaptadas a ambientes abertos, que podem então se estabelecer no fragmento (Lovejoy et al. 1984, 1986). 
A comunidade de aranhas apresenta grande sensibilidade a alterações ambientais que agem sobre a estrutura dos hábitats e sobre os fatores micro-climáticos a ele associados, causando modificações significativas no padrão de distribuição das espécies (Huhta 1971, Wise 1993). Apesar disto, existem algumas aranhas que podem tolerar flutuações dos fatores ambientais, em contraste com outras, que são menos flexíveis a essas mudanças, mostrando que cada espécie apresenta uma resposta diferente (Foelix 1996). Neste estudo foram encontradas algumas morfoespécies a exemplo de Tenedos sp. 1, e Oligoxystre sp. que parecem não sofrer efeitos de perturbação uma vez que ocorrem em todos os ambientes com abundâncias similares. Outras, como Lygromma sp. 1, Gamasomorphinae sp. 2, Alpaida sp., Neoxyphinus sp. 1, aff. Neonella sp. e Gamasomorphinae sp. 1, apresentaram maiores abundâncias nos ambientes de cabruca e capoeira, o que pode ser uma resposta oportunista, por possuírem a capacidade de habitar ambientes modificados. Por outro lado Linyphiidae sp. 1, Hahniidae sp. 1, Linothele sp., Salticidae sp. 12, Salticidae sp. 23 e Orchestina sp. 1 apresentam maiores abundâncias nos ambientes de mata e queda de abundância nos ambientes de cabruca e capoeira, o que pode ser uma resposta negativa à alteração ambiental, uma vez que essas morfoespécies possivelmente apresentam uma preferência por ambientes não modificados.

Não se constatou diferenças significativas ( $p>0,05)$ entre a composição de morfoespécies de aranhas de solo dos ambientes amostrados. As categorias ambientais definidas (matas contínuas, fragmentos de mata, cabrucas e capoeiras) podem não refletir os fatores ambientais que influenciam a comunidade de aranhas. Embora tenha se notado alterações na abundância individual de algumas morfoespécies que podem provocar a médio e a longo prazo modificações nessa comunidade. Estes resultados não significam que as aranhas de solo do Sul da Bahia sejam imunes a alterações na estrutura do ambiente. Ao contrário, a literatura é rica em exemplos de perda de espécies de artrópodes provocadas por substituição de ecossistemas naturais por equivalentes simplificados, como monocultivos ou pastagens, como o observado por Baert et al. (1991).

Deve-se salientar que os ambientes mais alterados (cabruca e capoeira), apesar da perturbação, se assemelham a uma floresta simplificada, que pode ser utilizada por diversos organismos florestais, sendo, portanto áreas importantes para a manutenção da araneofauna. Principalmente considerando o grande potencial biológico da Mata Atlântica, percebe-se a necessidade de manter os fragmentos florestais estudados, mesmo apresentando um certo grau de perturbação, conservando assim, a grande diversidade ainda existente.

\section{Agradecimentos}

A Universidade Estadual de Santa Cruz, em especial ao Curso de Pós-Graduação em Desenvolvimento Regional e Meio Ambiente, a Coordenação de Aperfeiçoamento de Pessoal de Nível Superior (CAPES) e IBAMA, por tornarem possível a realização deste trabalho. Ao Projeto Resta-Una pelo apoio nas coletas de campo. Aos pesquisadores: Rogério Bertani (Instituto Butantan) por identificar as Mygalomorphae, Adalberto José dos Santos e Walter Cerqueira pela leitura cuidadosa do manuscrito e críticas ao trabalho.

\section{Referências Bibliográficas}

ABRAHAM, B. J. 1983. Spacial and temporal patterns in a sagebrush steppe spider community (Arachnida: Araneae). The Jounal of Arachnology, v. 11, p. 31-50.

ARAUJO, M.; ALGER, K.; ROCHA, R.; MESQUITA, C. 1998. A Mata Atlântica do Sul da Bahia: Situação atual, ações e perspectivas. Caderno 08. Conselho Nacional da Reserva da Biosfera da Mata Atlântica. São Paulo, SP. $35 \mathrm{p}$

AYRES, M. ; JR. M. AYRES ; D. L. AYRES \&A. S. SANTOS. 2000. Biostat 2.0: Aplicações estatísticas nas áreas das ciências biológicas e médicas. Belém. Sociedade Cívil Mamirauá.

BAERT,L.; DESENDER, K.; MAELFAIT, J. P. 1991. Spider communieties of isla Santa Cruz (Galápagos, Equador). Journal of Biogeography, v. 18, p. 333-340.

COREY, D. T.; TAYLOR, W. K. 1988. Ground surface spiders in three central Florida plant communities. Journal of Arachnology, v.16, p. 213 - 221

BROWN, K. S. 1991. Conservation of Neotropical environments: Insects as indicators. In Collins, N.M. e J.A. Thomas (eds.). Conservation of insects and their natural environments.R.E.S. Symposium Nr. 15.Academic Press, London. 350-404. p

DEAN, W. 1996. A ferro e fogo: A história e a devastação da Mata Atlântica brasileira.Companhia das Letras. São Paulo, SP. 484 p.

FOELIX,R.F. Biology of Spiders. 1996. Second Edition. New York - Oxford. Oxford University Press. Georg Thieme Verlag. $330 \mathrm{p}$.

GASNIER, T. R.; HÖFER, H.; BRESCOVIT,A. D. 1995. Factors affecting the "activity density" of spiders on tree trunks in an Amazonian rainforest. Ecotropica, v.1, p. 269-277.

GREEN, J. 1999. Sampling method and time determines composition of spider collection. The Journal of Arachnology, v. 27, p. 176-182.

HAFFER, J. 1974. Avian speciation in tropical South America. Publication of the Nuttall Ornithological Club. v.14, p. 1390.

HUHTA, V. 1971. Sucecssion in the spiders communities of the floor after clear-cutting and prescribed burning. Ann. Zool. Fennici., v. 8, p. 483-542.

JACKSON, J. F. 1978. Differentiation in the genera Enyalius and Strobilurus (Iguanidae): Implications for Pleistocene climatic changes in eastern Brazil. Arquivos de Zoologia, v. 30, p. 1- 79 . 
JOCQUÉ, R. 1984. Considérations concernant l’ abondance relative des araignées errantes et des araignées à toile vivant au niveau du sol. Revue Arachonologique, v.5 (4), p. 193-204.

LAURANCE, W. F.; BIERREGAARD, R. O. Jr. 1997. Tropical forest remnants: Ecology, Managent,and Conservation of Fragmented Communities. The University of Chicago Press. Chicago \& London. p. 616

LOVEJOY, T. E.; A. B. RYLANDS; J. R. MALCOLM; K. S. BROWN JR. 1984. Ecosystem decay of Amazon forest remnants, p. 295-325. In: M. H. NITECKI. Extinctions. Chicago, University of Chicago.. p. 354

LOVEJOY, T.E; R. O. BIERREGAARDJR.;A. B. RYLANDS; J. R. MALCOLM; C. E. QUINTELA; L. H. HARPER; K. S. BROWN JR.;A.H. POWELL; H.O. R. SCHUBARTEM.B. HAYS. 1986. Edge and other effects on isolation on Amazon forest fragments. p.257-285. In: M.E. SOULE(Ed). Conservation biology: the science of scarcity and diversity. Sunderland, Massachusetts. Sinauer. 584 p.

MORI, S. A.; B. B. BOOM \& G. T. PRANCE. 1981. Distribution patterns and conservation of eastern Brazilian coastal forest tree species. Brittonia, v. 33, p. 233-245.

MYERS, N. 1980. Conversion of tropical moist forests. In National Research Council, National Academy of Science, Committee on Research Priorities (ed.). Tropical Biology. Washington D. C. 205 p.

NOSS, R. F. E B. CSUTI. 1994. Habitat fragmentation. In Meffe, K.G. \& C.R. Carroll (eds.) Principles of Conservation Biology. Sinauer Press.

ODUM, E. P. 1985. Ecologia. Interamericana, Rio de Janeiro $434 \mathrm{p}$.

PALMGREN, P.; BISTRÖM, O. 1979. Populations of Araneae (Arachoidea) and Staphylinidae (Coleoptera) on the floor of primeval forest in Mäntyharju, southern Finland Ann. Zool. Soc. Lond., v. 42, p. 131 - 138.

PIANKA, E. R. Ecologia Evolutiva. Omega, Barcelona, 366 pp. 1982

PIKELIN, B. G.; SCHIAPELLI, R.D. 1963. Llave para la determinacíon de familias de areñas argentinas. Physis. V. 24(67), p. 43-72.

PLATNICK, N. I. 2005. The world spiders catalog. version 5.5. By The American Museum of Natural History. Online at http:// reasearch.amnh.org/entomology/spiders/catalog/index.html.

POST III, W. M.; RIECHERT, S. E. 1977. Initial investigation in the structure of spiders communities. J. Anim. Ecol., v. 46, p. 729-749.

PRANCE, G. T. 1982. Forest refuges: evidence from woody Angiosperms. In: Prance, G.T. (editor). Biological diversification in the tropics. Columbia University Press, New York. 137-157p.

RICHARDSON, S. L.; LAROCHE, J. L. ; RICHARDSON, M. D. 1980. Larval fish assemblage and associations in north-east pacific ocean along the Oregon, coast winter-spring 1972-75. Estuarine and coastal Marine science, v. 11 , p. 671-699.
RICKLEFS, R. A economia da natureza. 1996. GuanabaraKoogan. Terceira edição. Cap. comunidades, p. 331-389. $470 \mathrm{p}$.

ROTH, M. 1993. Investigations on lead in the soil invertebrates of a forest ecosystem. Pedobiologia, 37: 270-279.

RYLANDS, A. B. 1982. The behaviour and ecology of three species of marmosets and tamarins (Callitrichidae, Primates) in Brazil. Tese de Doutorado. University of Cambridge, Cambridge, UK.

SCHVARTSMAN, S. 1992. Acidentes por aranhas (Araneísmo), 189-209. In: Plantas venenosas e animais peçonhentos. São Paulo. Sarvier. 271 p.

SIMÓ, M.; BRESCOVIT, A.D. 2001.Revision and cladistic analysis of the Neotropical spider genus Phoneutria PERTY, 1833 (Araneae, Ctenidae), with notes on the related Cteninae. Bull. Br. Arachnol.Soc., v. 12 (2), p. 67-82.

TERBAAK,C. J. F. ; SMILAUER, P. 1998. CANOCO Reference Manual and User's guide to Canoco for Windows. Microcomputer Power, Ithaca, USA, 35266.

THOMAS, W.W.; CARVALHO, A.V.; AMORIM,A.A.; GARRISON, J. \& ARBELÁEZ, A.L. 1998. Plant endemism in two forests in southern Bahia, Brazil. Biodiversity and Conservation, v. 7, p. 311-322.

TOTI, D. S.; COYLE, F.A.; MILLER, J.A. 2000. A structure inventory of Appalachian grass bald and heath bald spider assemblages and a test of species richness estimator performance. The Journal of Arachnology. V.28, p. 329-345.

TURNBULL, A. L. 1973. Ecology of the true spiders (Araneomorphae). Annu. Rev. Entomol, v. 18, p. 305 348.

UETZ, G. W. 1976. Gradient analysis of spider communities in streamside forest. Oecologia (Berl.), v.22, p. 373-385. .1979. The influence of variation in litter habitat on spider communities. Oecologia (Berl.), v. .40, p. 2942.

WISE, D. H. 1993. Spiders in ecological webs. New York. Cambridge University press. 328 p.

Título: Aranhas de solo (Arachnida: Araneae) em fragmentos florestais no sul da Bahia, Brasil.

Autores: Maria de Fátima da Rocha Dias, Antonio D. Brescovit \& Max de Menezes

Biota Neotropica, Vol. 5 ( número 1a): 2005

http://www.biotaneotropica.org.br/v5n1a/pt/ abstract?inventory+BN010051a2005

Recebido em 07/12/2003 - Publicado em 01/02/2005

ISSN 1676-0603 